\title{
Diversity of Planetary Atmospheric Circulations and Climates in a Simplified General Circulation Model
}

\author{
Yixiong Wang ${ }^{1}$ and Peter Read ${ }^{2}$ \\ ${ }^{1}$ Clarendon Laboratory, Department of Physics, University of Oxford, \\ Parks Road, OX1 3PU, Oxford, UK \\ email: y.wang1@physics.ox.ac.uk \\ ${ }^{2}$ Clarendon Laboratory, Department of Physics, University of Oxford, \\ Parks Road, OX1 3PU, Oxford, UK \\ email: p.read1@physics.ox.ac.uk
}

\begin{abstract}
The parametric dependence of terrestrial planetary atmospheric circulations and climates on characteristic parameters is studied. A simplified general circulation model-PUMA is employed to investigate the dynamic effects of planetary rotation rate and equator-to-pole temperature difference on the circulation and climate of terrestrial planetary atmospheres. Five different types of circulation regime are identified by mapping the experimental results in a 2-D parameter space defined by thermal Rossby number and frictional Taylor number. The effect of the transfer and redistribution of radiative energy is studied by building up a new two-band semi-gray radiative-convective scheme, which is capable of modelling greenhouse and anti-greenhouse effects while keeping the tunable parameters as few as possible. The results will provide insights into predicting the habitability of terrestrial exoplanets.
\end{abstract}

Keywords. Earth, hydrodynamics, radiatve transfer.

\section{Introduction}

The development of theories regarding the general circulation of planetary atmospheres has long been limited to Solar System planets (Williams \& Holloway 1982). With the discovery of more and more extrasolar planets in recent years, it is clear that we are now in an era when the study of comparative atmospheric circulations within a broader parameter range is possible (Read 2011, Showman et al. 2010). Due to limitations of the current observational techniques, only global/disk-integrated properties of exoplanets are available so far. In order to estimate the circulation structure and the consequential climate, our ability to predict properties of the circulation regime from bulk planetary parameters is sorely needed. However, a systematic study of the parametric dependence of global circulation regime on relavant planetary parameters is surprisingly missing in the atmospheric science literature, except in some early works by Williams (1988a), Williams (1988b), and Geisler et al. (1983). This paper aims to present some of our preliminary efforts towards building up a 'big picture' of planetary atmospheric circulations, in which different circulation regimes will be classified according to values of their characteristic parameters.

Inspired by laboratory rotating annulus experiments [which could be viewed in some respects as an analogue of a terrestrial atmosphere, see Hide \& Mason (1975)], we define two characteristic dimensionless parameters for planetary atmospheric circulations:

$$
\text { thermal Rossby number } \mathcal{R}_{o}=\frac{R \Delta \theta_{h}}{\Omega^{2} a^{2}},
$$


and

$$
\text { a frictional Taylor number } \mathcal{T}_{a}=4 \Omega^{2} \tau_{f}^{2},
$$

in which $R$ is the gas constant, $\Delta \theta_{h}$ the horizontal temperature difference between the equator and the poles, $\Omega$ the planetary rotation rate, $a$ the planetary radius, and $\tau_{f}$ the frictional timescale within the boundary layer.

\section{Description of the numerical model and experiments}

We use a simplified general circulation model(GCM)-PUMA (Portable University Model of the Atmosphere) developed by the University of Hamburg (Fraedrich et al. 2005). It has a pseudo-spectral dynamical core with linearized physical processes and $\sigma$-coordinate finite difference grid in the vertical direction. The diabatic heating and cooling is represented by Newtonian cooling, and the boundary layer frictional damping is represented by Rayleigh friction. We run the model with annual-mean thermal forcing, without topography.

Experiments are performed by specifying values for the rotation rate $\Omega\left(8 \Omega_{E}, 4 \Omega_{E}\right.$, $2 \Omega_{E}, 1 \Omega_{E}, 1 / 2 \Omega_{E}, 1 / 4 \Omega_{E}, 1 / 8 \Omega_{E}$ and $1 / 16 \Omega_{E}$, where $\Omega_{E}$ is the rotation rate of the Earth) as well as the imposed temperature difference $\Delta \theta_{h}(60 K, 30 K$, and $10 K)$ between the equator and the poles of the relaxation temperature field used in the Newtonian cooling scheme. There were 10 vertical levels for all experiments. Experiments with rotation rate higher than $1 \Omega_{E}$ were run with resolution of $\mathrm{T} 127$, and the others were run with T42 resolution. All experiments were run for 20 model years to ensure that the model atmosphere had spun up to an equilibrated state.

\section{Regime diagram}

A regime diagram was obtained by mapping the occurences of different circulation regimes observed within a parameter space spanned by thermal Rossby number $\mathcal{R}_{o}$ and frictional Taylor number $\mathcal{T}_{a}$ (see Fig. 1). Five different types of global circulation can be found in this regime diagram.

Axisymmetric flow. In our experiments, axisymmetric flows are found in the lower left region of the regime diagram, with slow rotation rate and small equator-to-pole temperature constrast. Axisymmetric circulations are featured by smooth laminar flows encircling the axis of planetary rotation, with no wave/eddy disturbances.

Cyclostrophic, super-rotating flow. This is the circulation regime that can be found on slowly-rotating planetary bodies in the Solar Sytem such as Venus and Titan. Due to the weakness of Coriolis force on these slow rotators, the major mechanical balance accounting for the large-scale atmospheric motion is the balance between pressure gradient force and the centrifugal force, which is known as cyclostrophic balance. Another unique feature of this kind of circulation is that there is very strong prograde wind over the equatorial regions, a phenomenon known as equatorial super-rotation (Read 1986). Figure 2 shows a cross-section of the zonal mean zonal wind averaged over the last 360 model days in the experiment with rotation rate $1 / 16 \Omega_{E}$, which shows clear evidence of super-rotation. The jet streams, which usually locate in mid-latitude or subtropics in Earth's atmosphere, are pushed further polewards due to the expanded Hadley cells under weak rotational constraints.

Regular baroclinic flow. For planets with an intermediate thermal Rossby number (larger than Earth's, but smaller than those of Venus and Titan), for which Mars may be an 


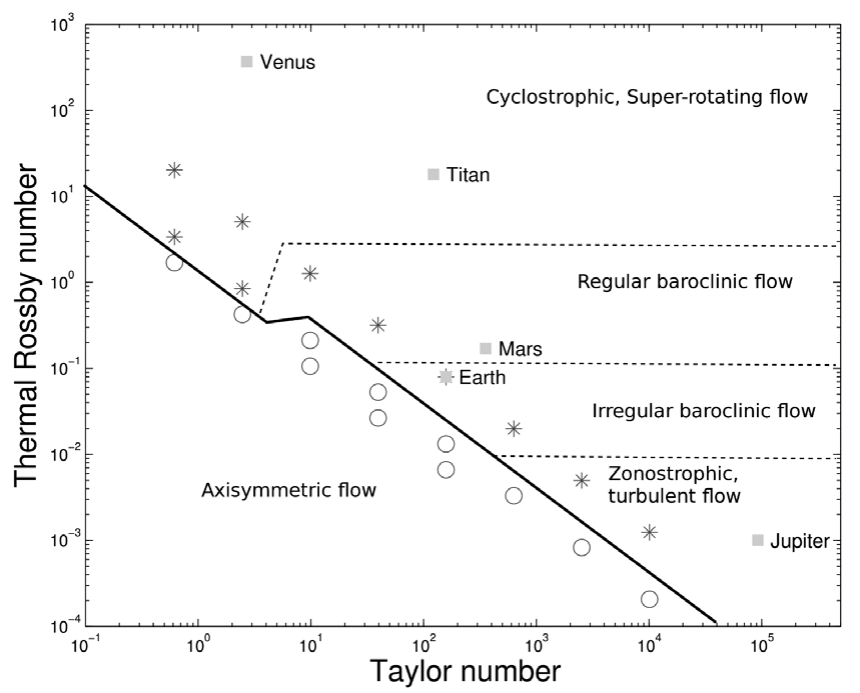

Figure 1. Regime diagram showing the various circulation regimes with respect to characteristic dimensionless parameters. Stars are experiments in which wavy flows are discovered, whereas circles are experiments in which axisymmetric flows are found. Some Solar System planets are labeled as squares. The solid line delineates the boundary between axisymmetric circulations and circulations with wavy/turbulent flows. The dashed lines are the boundaries between different circulation regimes within the wavy/turbulent region.

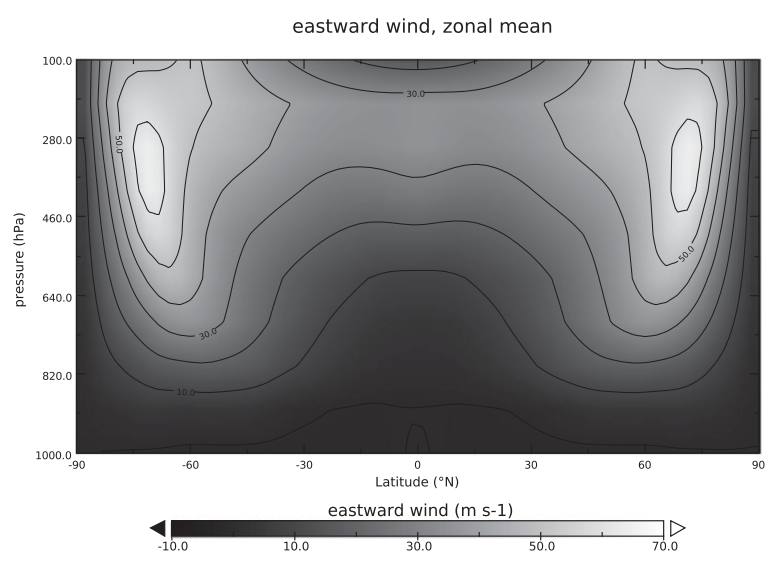

Figure 2. Zonal mean zonal wind for experiment with rotation rate of $1 / 16 \Omega_{E}$. Positive value refers to prograde direction.

example, the planetary baroclinic waves tend to be very regular and coherent in structure. Figure 3 shows a polar view snapshot of the geopotential height field in the experiment with rotation rate of $1 / 2 \Omega_{E}$. This experiment has roughly the same thermal Rossby number as that of Mars. A regular baroclinic wave with the dominating wavenumber-4 mode is found at $500 \mathrm{mb}$ level.

Irregular baroclinic flow. This is the type of general circulation represented by the Earth's atmosphere, characterised by irregular baroclinic waves with mixed wavenumbers.

Zonostrophic, turbulent flow. For planets with much smaller thermal Rossby number $\left(\overline{\left.\mathcal{R}_{o}<10^{-2}\right) \text {, the development }}\right.$ of zonostrophic turbulence will lead to the formation and 


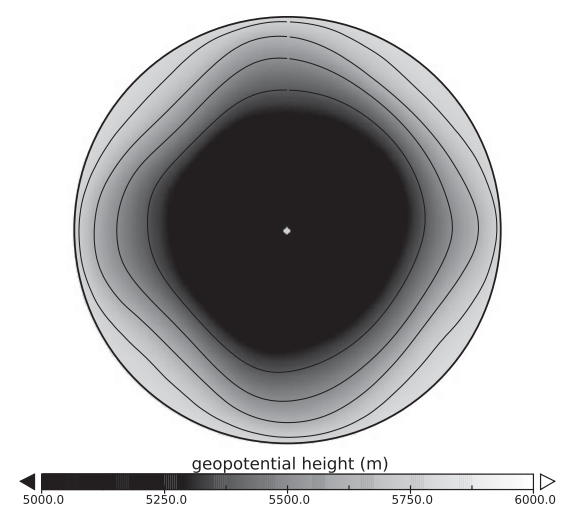

Figure 3. Snapshot of the geopotential height field at $500 \mathrm{mb}$ level for experiment with rotation rate of $1 / 2 \Omega_{E}$, viewed from above the north pole.

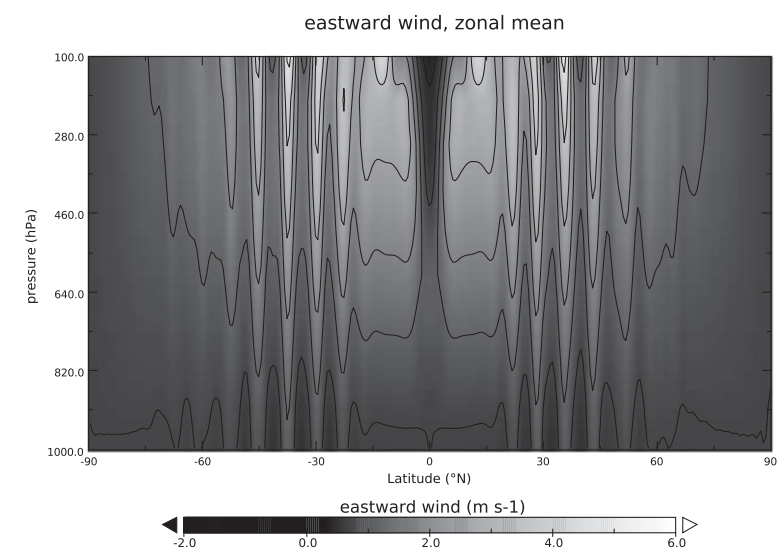

Figure 4. Zonal mean zonal wind for experiment with rotation rate of $8 \Omega_{E}$.

maintenance of multiple eddy-driven jets in the presence of strong rotation ( $\beta$-effect) and weak friction. Zonostrophic turbulence is a proposed mechanism for explaining the ubiquitous zonal jets discovered on Jovian planets as well as in the Earth's ocean (see Galperin et al. 2004). Figure 4 shows the zonal mean zonal wind cross-section of an experiment with about the same $\mathcal{R}_{o}$ as Jupiter.

\section{Parameter for radiative transfer}

The Newtonian cooling scheme in PUMA model requires an ad hoc relaxation temperature field, which is unable to give a physically realistic representation of radiative transfer processes in planetary atmospheres. A simple two-band semi-gray radiativeconvective scheme has therefore been developed to replace the Newtonian cooling scheme in PUMA. We assume that the stellar radiation and the planetary thermal radiation can be separated into two spectral bands (This assumption breaks down, for example, for close-in planets around brown dwarfs, in which case the temperature difference between the planet and its parent star is small). Plane parallel assumptions are made and radiatively active constituents of the atmosphere are assumed to be well-mixed in the vertical direction. The extinction coefficient is assigned a constant value in each band. A dry 

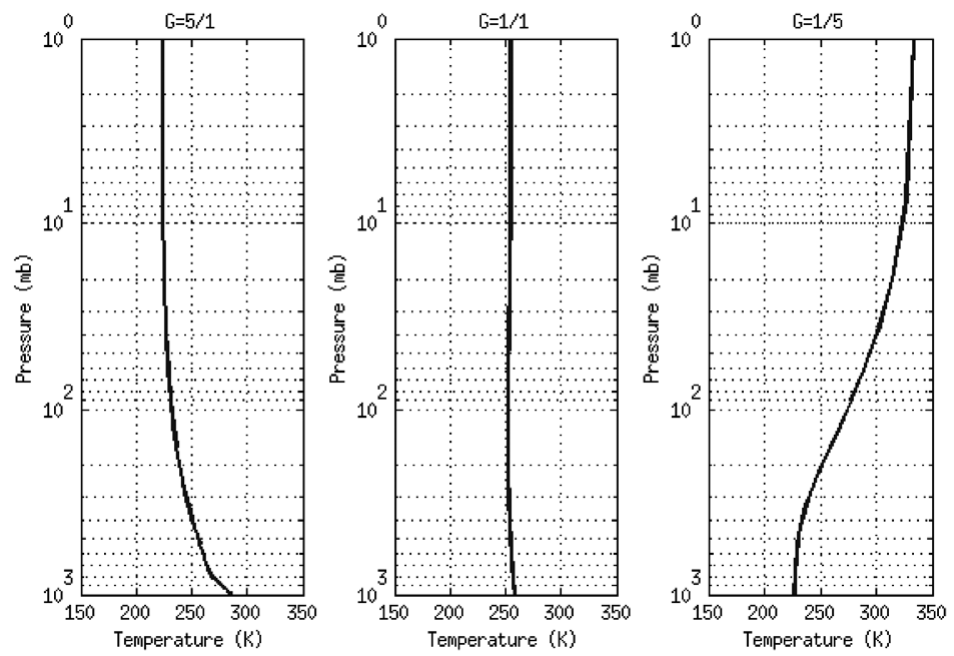

Figure 5. Temperature profiles with three different values of $G$, corresponding to greenhouse, neutral and anti-greenhouse scenarios. The quantity $\tau_{l w}=2.0$ for all plots.

convective adjustment scheme following Manabe \& Wetherald (1967) is employed to restore to a statically neutral temperature profile.

A parameter characterizing the transfer and distribution of radiative energy can be defined as $G=\tau_{l w} / \tau_{s w}$, where $\tau_{l w}$ is the total atmospheric optical depth in the long-wave (planetary thermal) band measured along the zenith direction, and $\tau_{s w}$ is the optical depth in the short-wave (stellar) band. The parameter $G$ can be essentially viewed as a greenhouse parameter which measures the intensity of the greenhouse effect of the atmosphere. If $G>1$ then the atmosphere is optically thick for the planetary thermal radiation but optically thin for the incoming stellar radiation, which means the temperature profile is mainly governed by a greenhouse effect (cf. Earth, Venus, Mars). If $G<1$ then the atmosphere is optically thin in the long-wave thermal band, but relatively thick in the short-wave stellar band, which means the atmosphere is mainly heated by downwelling stellar radiation. This will lead to a more stable temperature profile with warm upper atmosphere and cold lower atmosphere (See Fig. 5), which is sometimes referred to as an 'anti-greenhouse' effect (McKay et al. 1991).

\section{Future work and Discussions}

At the time of writing, the two-band radiative-convective scheme had been coupled with the dynamic core of the original PUMA model, leading to a new version of PUMA (PUMA-G). By replacing the Newtonian cooling scheme with this more realistic radiativeconvective scheme, more defining parameters related to the radiative transfer processes should be taken into consideration. Apart from the greenhouse parameter $G$, the obliquity of the planetary rotation axis is also very important in shaping the radiative-equilibrium temperature field. Therefore we are planning to build up a 3-D parameter space for PUMA-G spanned by thermal Rossby number $\mathcal{R}_{o}$, greenhouse parameter $G$ and the obliquity $\delta$.

By putting our study into the context of exoplanet characterizations, we propose a preliminary list of planetary parameters to be observed and constrained by astronomers in order to determine the possible circulation and climate regimes of a certain exoplanet. This includes parameters like planetary size, distance between the planet and the 
parent star, planetary rotation rate, obliquity and radiative properties of the atmosphere, although some of them are still challenging for current observational techniques. By carefully constraining these parameters, we can in principle derive aspects of the corresponding circulation regimes based on our regime diagram studies.

\section{References}

Fraedrich, K., Kirk, E., Luksch, U., \& Lunkeit, F. 2005, Meteorol. Z., 6, 14

Galperin, B., Nanako, H., Huang, H-P., \& Sukoriansky, S. 2004, Geophys. Res. Lett, 13, 31

Geisler, J. E., E. J. Pitcher, \& R. C. Malone 1983, J. Geophys. Res., 88

Hide, R. \& Mason, P. 1975, Adv. Phys, 1,24

Manabe, S. \& Wetherald, R. T. 1967, J. Atmos. Sci, 24

McKay, C. P., Pollack, J. B., \& Courtin, R. 1991, Science, 253

Read,P. 1986, Quart. J. R. Met.Soc., 112

Read, P. 2011, Planet. Space. Sci., 10, 59

Showman, A. P., Cho, J. Y.-K., \& Menou, K. 2010, in: Seager, S.(eds.), Exoplanets (University of Arizona Press, Tucson, Arizona)

Williams,G. P. \& Holloway, J. L. 1982, Nature, 297

Williams, G. P. 1988, Clim. Dynam. I, 2, 205

Williams, G. P. 1988, Clim. Dynam. II, 3, 45 